\title{
Linear theory of non-neutral plasma equilibrium in a tilted magnetic field
}

\author{
Ross L. Spencer \\ ross_spencer@byu.edu \\ Grant W. Hart
}

Follow this and additional works at: https://scholarsarchive.byu.edu/facpub

Part of the Astrophysics and Astronomy Commons, and the Physics Commons

\section{Original Publication Citation}

Spencer, R. L. and G. W. Hart."Linear theory of non-neutral plasma equilibrium in a tilted magnetic field." Physics of Fluids B: Plasma Physics 4 (1992): 357-3512.

\section{BYU ScholarsArchive Citation}

Spencer, Ross L. and Hart, Grant W., "Linear theory of non-neutral plasma equilibrium in a tilted magnetic field" (1992). Faculty Publications. 712.

https://scholarsarchive.byu.edu/facpub/712 


\title{
Linear theory of non-neutral plasma equilibrium in a tilted magnetic field
}

\author{
R. L. Spencer and G. W. Hart \\ Department of Physics and Astronomy, Brigham Young University, Provo, Utah 84602
}

(Received 3 January 1992; accepted 23 June 1992)

\begin{abstract}
A linear perturbation expansion has been found that allows the rapid and accurate calculation of the response of a non-neutral plasma to a tilted magnetic field. The results of the calculation have been found to agree with previous three-dimensional equilibrium calculations, and also to agree with Keinigs' [Phys. Fluids 24, 860 (1981)] calculation of zerofrequency resonances caused by magnetic field errors. This expansion also allows the perturbed velocity to be calculated. It is speculated that this perturbed flow may be related to the enhanced radial transport in a non-neutral plasma with a tilted magnetic field.
\end{abstract}

\section{INTRODUCTION}

It is well known that non-neutral plasmas are sensitive to various kinds of magnetic and electric field errors. ${ }^{1-4} \mathrm{~A}$ particularly simple example of such an error is the tilting of the confining magnetic field with respect to the axis of the conducting cylinder. A recent study of this misalignment of the magnetic field used a three-dimensional equilibrium calculation to compute the nonlinear coupling between the magnetic field perturbation and the $l=1$ diocotron mode and compared that result to experimental data. ${ }^{5}$ That three-dimensional calculation, however, was very slow and too coarse to reveal small, but interesting, features such as equilibrium flow. This paper attempts to remedy this situation by using a linear perturbation expansion in the small tilt angle $\alpha$ to obtain two-dimensional equations for the deviation of the plasma from axisymmetric equilibrium. The resulting equations are solved numerically, and the solutions are found to agree very well with the results of the equilibrium solver of Ref. 5. In addition, the equations indicate that the tilted plasma is required to have flow. If this flow were to become unstable and cause mixing, it might account for the observed flattening of density profiles when such a field misalignment is purposely produced. ${ }^{6}$ We also discuss the connection of this calculation to Keinigs' calculation of resonant magnetic field errors ${ }^{7}$ and find in full two-dimensional geometry the same effects he found in infinitely long geometry.

\section{PERTURBED EQUILIBRIUM}

Consider a cylindrically symmetric non-neutral plasma contained within a conducting cylinder of radius $a$ and radially confined by a magnetic field $\mathbf{B}=B_{0} \hat{z}$. The plasma is made up of particles of mass $m$ and charge $q$, and is modeled as a fluid with constant temperature $T$. With these assumptions the equilibrium equations are

$$
\nabla \cdot n_{0} \mathbf{v}_{0}=0,
$$

and

$$
n_{0} m \mathbf{v}_{0} \cdot \nabla \mathbf{v}_{0}=-n q \nabla \phi_{0}-k T \nabla n_{0}+q n_{0} \mathbf{v}_{0} \times \mathbf{B}
$$

where the equilibrium density, electric potential, and flow velocity are given by

$$
n_{0}(r, z), \quad \phi_{0}(r, z), \quad \nabla_{0}=r \omega_{0}(r, z) \hat{\theta} .
$$

The equilibrium rotation frequency $\omega_{0}$ can be calculated from the radial component of the fluid momentum equation, Eq. (2) (assuming that inertial effects are small) to be

$$
\omega_{0}(r, z)=\omega_{E}+\omega_{D}=\frac{1}{r B_{0}}\left(\frac{\partial \phi_{0}}{\partial r}+\frac{k T}{q n_{0}} \frac{\partial n_{0}}{\partial r}\right),
$$

where $\omega_{E}$ is the $\mathbf{E} \times \mathbf{B}$ drift frequency and where $\omega_{D}$ is the diamagnetic drift frequency. The equilibrium diamagnetic drift does not influence the plasma perpendicular to the magnetic field, but radial pressure gradient effects do play a role in the inertial response of the plasma along the tilted field, as noted later.

A typical non-neutral plasma equilibrium, without a tilted field, is displayed in Fig. 1. This particular plasma is an electron plasma in thermal equilibrium (rigid rotor) with a maximum density of $5 \times 10^{12} \mathrm{~m}^{-3}$, a temperature of $1 \mathrm{eV}$, and is confined by end potentials of $175 \mathrm{~V}$ in a cylinder of radius $4 \mathrm{~cm}$.

The magnetic field is tilted by adding a small amount of magnetic field in the $y$ direction:

$$
\mathbf{B}=B_{0} \hat{\mathbf{z}}+\alpha B_{0} \hat{\mathbf{y}},
$$

where $\alpha$ is a small number. When $\alpha$ is small, it is approximately the tilt angle of the magnetic field. We now seek a steady-state solution of the following equations, to first order in $\alpha$ :

continuity

$$
\boldsymbol{\nabla} \cdot \boldsymbol{n v}=\mathbf{0}
$$

drift motion perpendicular to $\mathbf{B}$

$$
\mathbf{v}_{\perp}=(-\nabla \phi \times \hat{\mathbf{b}}) / B_{0},
$$

where $\hat{\mathbf{b}}$ is a unit vector in the direction of the tilted magnetic field:

$$
\widehat{\mathbf{b}}=(\hat{\mathbf{z}}+\alpha \hat{\mathbf{y}}) /\left(1+\alpha^{2}\right)^{1 / 2} .
$$




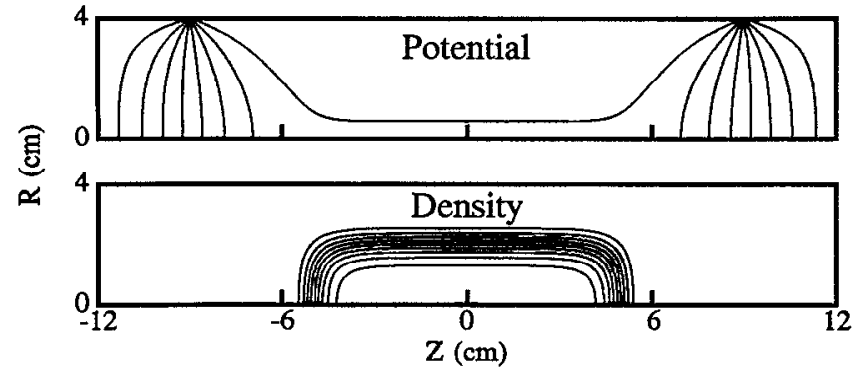

FIG. 1. Equilibrium potential and density for a $1 \mathrm{eV}$ thermal equilibrium plasma. The potential contour increment is $20 \mathrm{~V}$ and the density increment is $5 \times 10^{11} \mathrm{~m}^{-3}$.

Note that the diamagnetic drift has been ignored here in spite of finite temperature because the divergence of the mass flux from this drift velocity is zero, and hence it can have no influence on the perpendicular part of the tilted equilibrium. In this calculation, finite temperature and inertial effects only influence motion parallel to the magnetic field:

\section{Parallel momentum}

$$
m n \hat{\mathbf{b}} \cdot \mathbf{v} \cdot \nabla \mathbf{v}=-q n \hat{\mathbf{b}} \cdot \nabla \phi-k T \hat{\mathbf{b}} \cdot \nabla n,
$$

where $n$ is the particle density, $\phi$ is the electric potential, $\mathbf{v}$ is the fluid velocity, and where $k T$ is the temperature in energy units (assumed constant and uniform).

\section{Poisson's equation}

$$
\nabla^{2} \phi=\left(-q / \epsilon_{0}\right) n \text {. }
$$

We now make a linear expansion in the small tilt angle $\alpha$ by writing

$$
\phi=\phi_{0}+\phi_{1}, \quad n=n_{0}+n_{1}, \quad \mathbf{v}=\mathbf{v}_{0}+\mathbf{v}_{1},
$$

where $\phi_{1}, n_{1}$, and $v_{1}$ are the linear corrections to the equilibrium quantities caused by the tilt. We also linearize $\mathrm{Eq}$. (8):

$$
\hat{\mathbf{b}} \approx \hat{\mathbf{z}}+\alpha \hat{\mathbf{y}} \text {. }
$$

We choose to carry out this expansion in cylindrical coordinates aligned with the conducting cylinder, rather than aligned with the magnetic field. We first note that the diamagnetic drift velocity contributes nothing to the continuity equation because $\nabla \cdot n \mathbf{v}_{D}=0$, where $\mathbf{v}_{D}=-(\nabla p \times \hat{\mathbf{b}}) /(n B)$. We then write the continuity equation in the form

$$
\nabla \cdot n \mathbf{v}=n \nabla \cdot \mathbf{v}+\mathbf{v} \cdot \nabla n=0 .
$$

The divergence of the $\mathbf{E} \times \mathbf{B}$ drift velocity is zero, so we obtain

$$
n \partial_{\|} v_{\|}+\mathbf{v} \cdot \nabla n=0,
$$

where $\partial_{\|}$is the derivative along the direction of the magnetic field and where $v_{\|}$is the component of the fluid velocity along the magnetic field. This equation is now linearized to obtain

$$
\frac{\partial\left(n_{0} v_{1 z}\right)}{\partial z}+v_{1 r} \frac{\partial n_{0}}{\partial r}+\omega_{E} \frac{\partial n_{1}}{\partial \theta}=-\alpha r n_{0} \frac{\partial \omega_{E}}{\partial z} \cos \theta
$$

where in deriving this equation we have replaced $v_{\|}$with $v_{1 z}$ by using the linearized form of Eq. (7) to obtain

$$
v_{1 z}=v_{\|}-\alpha r \omega_{E} \cos \theta \text {. }
$$

Although the pressure gradient was not important in the continuity equation, it is important in the linearized parallel momentum equation:

$$
m \omega_{0} \frac{\partial v_{1 z}}{\partial \theta}+q \frac{\partial \phi_{1}}{\partial z}+k T \frac{\partial}{\partial z}\left(\frac{n_{1}}{n_{0}}\right)=\alpha \sin \theta F_{0}
$$

where $F_{0}$ is the combined radial electric and pressure gradient force in the untilted equilibrium:

$$
F_{0}=-q \frac{\partial \phi_{0}}{\partial r}-\frac{k T}{n_{0}} \frac{\partial n_{0}}{\partial r} .
$$

Note that the term containing $F_{0}$ in Eq. (17) is simply the component of the radial force along the new parallel direction in the tilted field. It appears because the Lorentz force can no longer balance this component of the force, as it did in the axisymmetric equilibrium. Note also that a small inertial contribution to $F_{0}$ has been neglected. Finally, we have Poisson's equation:

$$
\nabla^{2} \phi_{1}=-\left(q / \epsilon_{0}\right) n_{1} .
$$

To first order in $\alpha$, the tilt is just an $l=1$ perturbation, but since we have chosen the transverse component of the magnetic field to be in the $y$ direction, we must carefully choose the phases of the perturbed quantities. Equations $(10)-(12)$ require that we write

$$
\begin{aligned}
& \phi_{1}=\phi_{1}(r, z) \sin \theta, \quad n_{1}=n_{1}(r, z) \sin \theta, \\
& v_{1 z}=v_{1 z}(r, z) \cos \theta .
\end{aligned}
$$

With these choices Eq. (7) may be used to obtain the formula for $v_{1 n}$

$$
v_{1 r}=\left(\frac{-\phi_{1}}{r}+\alpha \frac{\partial \phi_{0}}{\partial z}\right) \frac{\cos \theta}{B_{0}},
$$

we solve Eq. (17) for $v_{1 z}$, then substitute in Eq. (15), and use Eq. (18) to obtain
(1)

$$
\begin{aligned}
& \frac{\partial}{\partial z}\left(\frac{n_{0}}{\omega_{0}} \frac{\partial f}{\partial z}\right)+\frac{\partial}{\partial z}\left(\frac{n_{0}}{\omega_{0}} \frac{\partial h}{\partial z}\right)+\alpha \frac{q r B_{0}}{k T} \frac{\partial n_{0}}{\partial z}-\frac{m}{r q B_{0}} \frac{\partial n_{0}}{\partial r} \\
& \text { Phys. Fluids B, Vol. 4, No. } 11, \text { November } 1992
\end{aligned}
$$

(6) 


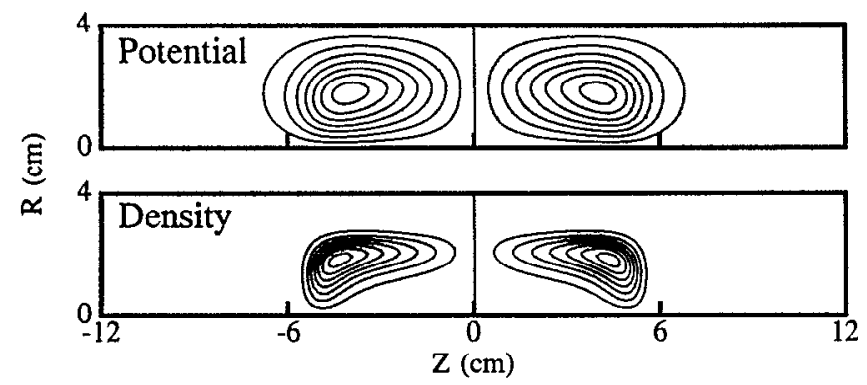

FIG. 2. Perturbed potential and density for the $1 \mathrm{eV}$ thermal equilibrium plasma shown in Fig. I when the magnetic field is tilted by $2.5 \times 10^{-3} \mathrm{rad}$. The potential contour increment is $7.8 \times 10^{3} \mathrm{~V}$ and the density increment is $9.3 \times 10^{9} \mathrm{~m}^{-3}$. The shaded regions indicate where the perturbed quantity is negative.

where

$$
f=q \phi_{1} / k T ; \quad h=n_{1} / n_{0} .
$$

This gives one relation between the perturbed potential and density, and Poisson's equation gives another. Together they determine the solution of the problem. It is possible simply to solve this set of equations numerically, and interesting resonance effects in plasmas near the Brillouin limit can be found in this way, as discussed in Sec. IV. But, for typical non-neutral plasmas, it is found that terms (4)-(7) are much smaller than terms (1)-(3). Hence we may approximately solve this equation by keeping only the first three terms. After solving for $f$ and $h$, we verify that terms (4)-(7) are indeed small, then use Eq. (15) approximately to solve for $v_{1 z}$. The resulting velocities are discussed in Sec. III. With these approximations we obtain

$$
h=-f+\frac{\alpha}{k T} \int_{0}^{z} F_{0} d z
$$

and

$$
\frac{1}{r} \frac{\partial}{\partial r} r \frac{\partial f}{\partial r}-\frac{f}{r^{2}}+\frac{\partial^{2} f}{\partial z^{2}}-k_{D}^{2} f=-\alpha k_{\mathrm{D}}^{2} \frac{1}{k T} \int_{0}^{z} F_{0} d z
$$

where $k_{\mathrm{D}}$ is the reciprocal of the local Debye length in the untilted equilibrium,

$$
k_{\mathrm{D}}^{2}=\frac{q^{2} n_{0}(r, z)}{\epsilon_{0} k T}
$$

(We have assumed that the untilted equilibrium is symmetric about $z=0$.) It is now a simple matter to use a standard Poisson solver with the modified operator of Eq. (25) to find the perturbed potential function, $f,(f=0$ on the conducting cylinder) and then to use Eq. (24) to obtain the perturbed density. Figure 2 shows contours of perturbed potential and density for the thermal equilibrium of Fig. 1 with a tilt angle of $\alpha=0.0025$. The neglected terms in Eq. (22) are typically found to be three orders of magnitude smaller than the included terms for a temperature of $1 \mathrm{eV}$.

\section{A. Central analytic solution}

If the plasma is much longer than a plasma diameter, it is possible to find a solution of Eq. (25) valid in the straight central region of the plasma. In this region, the right-hand side of Eq. (25) is proportional to $z$; if we assume that both $f$ and $h$ are also proportional to $z$, the second derivative with respect to $z$ vanishes and we simply have a radial ordinary differential equation to solve. If the following equation for $f$ is substituted into Eq. (25), it can be verified that it is a solution:

$$
f(r, z)=-\alpha z \frac{q \partial \phi_{0}(r, 0)}{k T \quad \partial r}+\alpha z f_{h}(r)
$$

where $f_{h}$ satisfies the homogeneous differential equation

$$
\frac{1}{r} \frac{d}{d r} r \frac{d f}{d r}-\frac{f}{r^{2}}-k_{\mathrm{D}}^{2}(r, 0) f=0 .
$$

The proof of this statement requires the use of Poisson's equation for a very long non-neutral plasma with central density profile $n_{0}(r, 0)$. The value of $f_{h}$ at the conducting wall, $r=a$, is chosen to make $f$ in Eq. (27) zero there:

$$
f_{h}(a)=\left.\frac{q}{k T} \frac{\partial \phi_{0}(r, 0)}{\partial r}\right|_{r=a} .
$$

This solution for $f$ in the central region may then be used in Eq. (24) to obtain the approximate perturbed density in this region:

$$
n_{1} \approx-\alpha z \frac{\partial n_{0}(r, 0)}{\partial r}-n_{0} \alpha z f_{h}(r)
$$

Note that the first terms in these forms for $n_{1}$ and $\phi_{1}$ [Eqs. (27) and (30)] are simply the expressions we would obtain by assuming that the equilibrium plasma has rotated about the $x$ axis by angle $\alpha$; e.g., $n_{1} \approx-\xi \cdot \nabla n_{0}, \xi=\alpha z \hat{y}$, as discussed in Ref. 5 . Hence the second term, which comes from the part of the solution for the perturbed potential which depends on the conducting wall, is just the perturbation produced by the image charge on the wall. Figure 3 shows the difference between the perturbed density computed by numerically solving Eq. (24) and that given by Eq. (30) for the equilibrium of Fig. 1. Note that they only disagree near the ends of the plasma. [In making this comparison, we chose to use Eq. (30) with $n_{0}(r, 0)$ replaced by $n_{0}(r, z)$. These two are identical in the straight central region, but the second form has the advantage that it goes to zero beyond the ends of the plasma, as does the actual perturbed density. This improves the approximation somewhat.]

Finally, Fig. 4 shows the difference between the perturbed density computed from Eqs. (24) and (25) and that computed by the three-dimensional equilibrium calculation of Ref. 5. The agreement is quite good $(2 \%-4 \%)$ except near the ends of the plasma. This disagreement is of approximately the same magnitude as the accuracy of the three dimensional equilibrium calculation because of the finite grid size. The disagreement near the ends of the plasma is probably exaggerated by the contour plot be- 


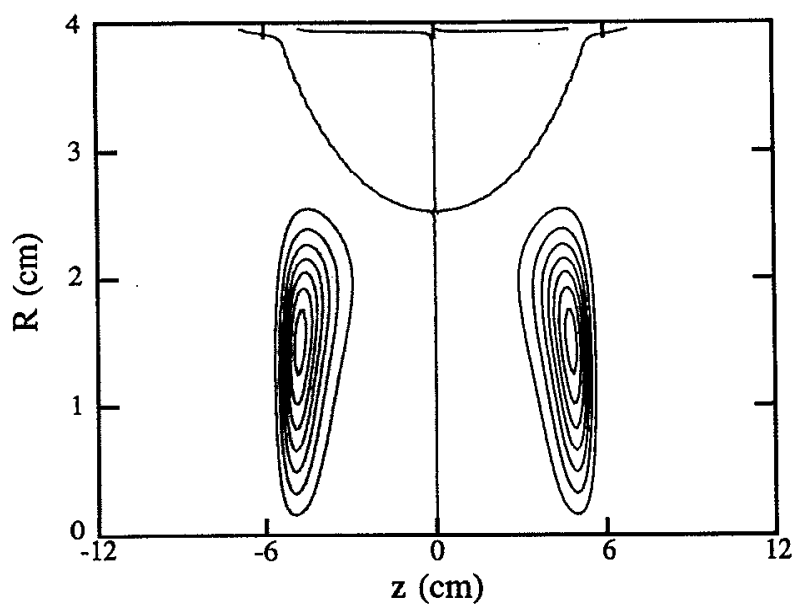

FIG. 3. Density difference between the solution of Eq. (24) and the analytic central approximation [Eq. (30)]. The contour increment is $4.4 \times 10^{9} \mathrm{~m}^{-3}$. Note that the analytic approximation accounts for virtually all of the perturbed density in the central regions of the plasma but only approximately $50 \%$ of the perturbed density at the ends. The shaded regions indicate where the difference is negative.

cause both calculations have steep gradients near the end, turning slight differences in position into large differences in density. When the peak values of the perturbed density in the two calculations are compared, they differ only by 7\%. This disagreement between the two calculations is of about the same magnitude and is concentrated in the same spatial regions as the nonlinear axisymmetric and $l=2$ terms in the three-dimensional equilibrium calculation. This may indicate that the assumption of linearity is just starting to break down in this end region.

Hence this method provides a fairly simple and accurate way to compute the response of typical non-neutral plasmas to tilted magnetic fields, and even provides an

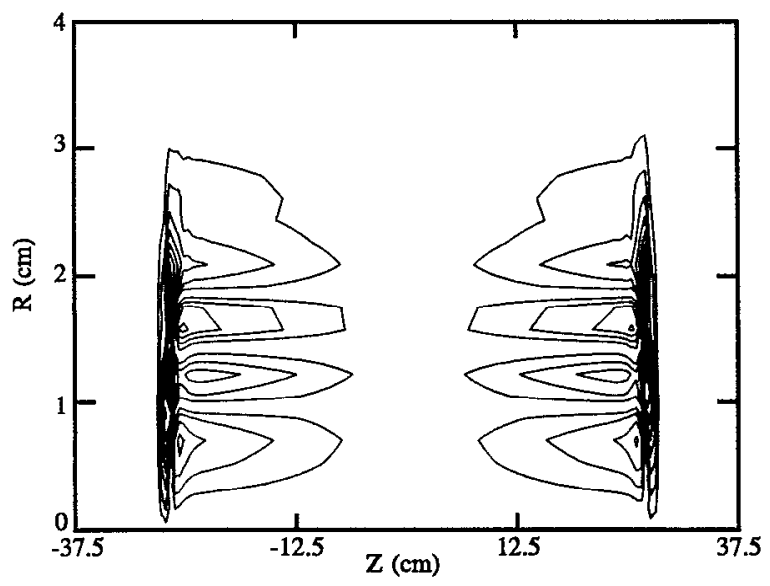

FIG. 4. Magnitude of the relative difference between the solution of Eq. (25) and the results of a three-dimensional equilibrium calculation. The contour increment is $2.5 \%$ relative difference. The larger values at the ends are probably due to the very large gradients in $z$ in both calculations. Small differences in position can translate into large $(\approx 20 \%)$ differences in perturbed density. analytic approximation valid in the central section. In addition, it also allows us to calculate the flow induced in the plasma by the tilt, as discussed in Sec. III.

\section{FLOW IN THE TILTED EQUILIBRIUM}

Because of grid size limitations in the threedimensional calculations described in Ref. 5, it was not possible to find the flow induced in the plasma by the tilted field. In this calculation, however, the flow may easily be obtained from the perturbed potentials and densities. We use Eq. (7) to obtain the perturbed flow perpendicular to the tilted magnetic field and Eq. (15) to obtain the flow parallel to the field. When these velocities are expressed in cylindrical coordinates aligned with the conducting cylinder, we have

$$
\begin{aligned}
v_{1 r}= & \left(\frac{-\phi_{1}(r, z)}{r}+\alpha \frac{\partial \phi_{0}}{\partial z}\right) \frac{\cos \theta}{B_{0}}, \\
v_{1 \theta}= & \left(\frac{\partial \phi_{1}(r, z)}{\partial r}-\alpha \frac{\partial \phi_{0}}{\partial z}\right) \frac{\sin \theta}{B_{0}}, \\
v_{1 z}= & \frac{\cos \theta}{n_{0}} \int_{-\infty}^{z}\left(\frac{\phi_{1}(r, z)}{r B_{0}} \frac{\partial n_{0}}{\partial r}-\omega_{E} n_{1}(r, z)\right. \\
& \left.-\frac{\alpha}{B_{0}} \frac{\partial \phi_{0}}{\partial z} \frac{\partial n_{0}}{\partial r}\right) d z-\alpha r \omega_{E} \cos \theta .
\end{aligned}
$$

Figure 5 displays these three components of the perturbed flow for the thermal equilibrium of Fig. 1. [Note that the plots are actually plots of velocity multiplied by the weighting factor $n_{0}(r, z) / n_{0}(0,0)$. This weighting was used to suppress the annoying large velocities which always appear in the vacuum regions in fluid calculations.] The unperturbed $\mathbf{E} \times \mathbf{B}$ flow velocity, $\mathbf{v}_{E}=r \omega_{E} \hat{\theta}$, is much larger than these perturbed velocities. Because of this, when the flow pattern is integrated to find the new flow streamlines, the deviation of a fluid element from the tilted field line is of the order of $\alpha z$ at the ends, and much smaller in the central section. This deviation is thus greater for large tilt angles and for long plasmas. The component of the flow that moves fluid elements across the magnetic field is concentrated near the ends of the plasma. This suggests that this equilibrium flow might have something to do with the observed slow flattening of density profiles in tilted plasmas. ${ }^{6}$ Perhaps the flow induced by the tilt is unstable, or is particularly susceptible to electric or magnetic perturbations, leading to enhanced transport toward thermal equilibrium. This is only speculation at this point, however; much further study of the dynamics of tilted plasmas will be required before anything more definitive can be said.

\section{RESONANCE EFFECTS}

As discussed by Keinigs ${ }^{7}$ and as observed by Heinze, ${ }^{4}$ field errors, such as the tilt discussed here, can resonate with low-frequency waves. From this point of view our tilted equilibrium, for proper choices of the parameters, should be a zero-frequency mode with very large perturbed 

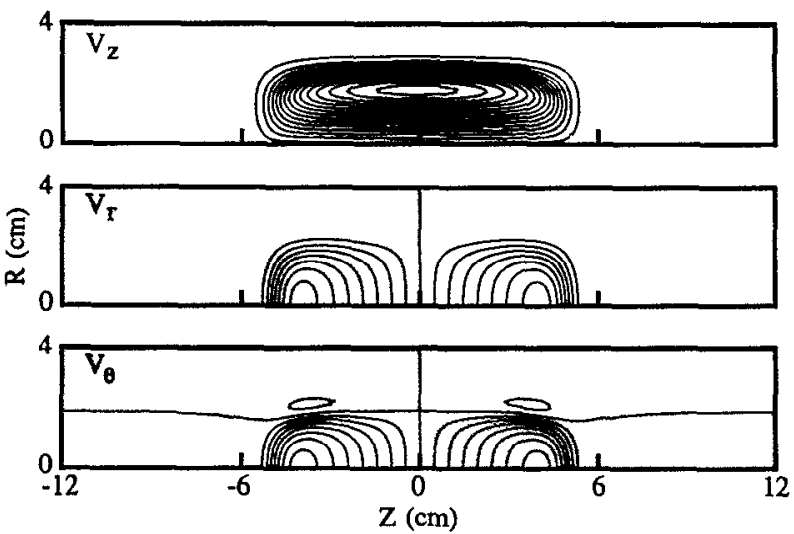

FIG. 5. Perturbed velocity for the equilibrium shown in Fig. 1 with the magnetic field tilted by $2.5 \times 10^{-3} \mathrm{rad}$. The contour increment is 9.8 $\mathrm{m} / \mathrm{sec}$ in the $V_{z}$ plot, $7.7 \mathrm{~m} / \mathrm{sec}$ in the $V_{r}$ plot, and $7.7 \mathrm{~m} / \mathrm{sec}$ in the $V_{\theta}$ plot. The regions where the velocity is negative are shaded. Here, $V_{z}$ and

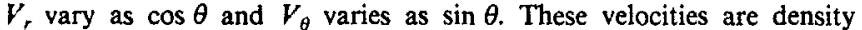
weighted [i.e., multiplied by $n_{0}(r, z) / n_{0}(0,0)$ ]. This has a very small effect in the interior of the plasma, but it causes the calculated velocity to be zero in the regions where there is no plasma.

fields and densities. This possibility is excluded from the calculations of Sec. II because of the terms we neglected there. To remedy this situation we must keep all terms in Eq. (22) and solve it simultaneously with Eq. (19). We use Eq. (19) to eliminate the perturbed density, $n_{1}=n_{0} h$, from Eq. (22) to obtain a partial differential equation for the perturbed potential, which is second order in $r$ and fourth order in $z$. We solve this equation by finite differencing it on an $r-z$ grid of size $n r \times n z$, and by using a banded matrix solver to find the perturbed potentials at the grid points. This is not just one choice among many possible ways to solve this problem; the equation has the nasty feature that it is elliptic over part of the computational region and hyperbolic over the other part, rendering many methods, such as iteration, useless.

As a check, we first verified that the numerical solution of the full Eq. (22) agreed with the approximate solution of Sec. II when the neglected terms were small. For the grids we used (typically $n r=20$ and $n z=150$ ), the differences between the two calculations were about $0.1 \%$. As we explored lower temperatures, we found that it was important to have the grid spacings $\Delta r$ and $\Delta z$ no larger than a Debye length.

With some confidence in the numerical method, we went looking for resonances. As a guide to where to look, we used Keinigs' approximate result that there should be a "finite- $k$ diocotron" mode for a magnetic perturbation of wave number

$$
k \approx \sqrt{2} \omega_{p} / a \omega_{c},
$$

where $a$ is the radius of the conducting wall, $\omega_{p}$ is the plasma frequency, and $\omega_{c}$ is the cyclotron frequency. We cannot make direct contact with Keinigs' calculation because a tilted magnetic field is a perturbation with infinite wavelength. However, the formula for $k$ given above de- pends on the appearance of $k$ in a term in the continuity equation which is proportional to

$$
\frac{\partial}{\partial z} n_{0} \delta B_{r}
$$

where $\delta B_{r}$ is the radial component of the magnetic perturbation. For Keinigs, $n_{0}$ is uniform in $z$ while $\delta B_{r}$ is proportional to $e^{i k z}$, giving for this term

$$
\frac{\partial}{\partial z} n_{0} \delta B_{r}=i k \delta B_{r} n_{0}
$$

In our calculation $\delta B_{r}$ is uniform in $z$, but $n_{0}$ depends on length, giving

$$
\frac{\partial}{\partial z} n_{0} \delta B_{r}=\delta B_{r} \frac{\partial n_{0}}{\partial z}
$$

For a long plasma $\partial n_{0} / \partial z$ has a bump at one end and a bump of opposite sign at the other end, looking sort of like a half-period of a sine wave of wavelength $4 z_{p}$, where $z_{p}$ is the half-length of the plasma. We thus make the crude estimate $k \approx \pi / 2 z_{p}$ to obtain from Eq. (33) the approximate resonance condition

$$
\omega_{c} / \sqrt{2} \omega_{p} \approx 2 z_{p} / \pi a \text {. }
$$

This condition has been written in such a way that the quantity on the left is a measure of how close the plasma is to the Brillouin limit, since at the Brillouin limit $\omega_{p}=\omega_{\mathrm{d}}$ $\sqrt{2}$. Equation (37) predicts that resonance in a short plasma would require that it be near the Brillouin limit, while longer plasmas need not be so close to the limit.

With these results as a guide, we looked at several different equilibria and slowly changed the magnetic field in the neighborhood of the Brillouin limit looking for resonances. Figure 6 shows the peak perturbed density, as a fraction of the peak equilibrium density, for more than 3000 different values of the magnetic field. The equilibrium we used for this calculation had a temperature of $1 \mathrm{eV}$ and plasma radius and half-length ratios of $r_{p} / a=0.5$ and $z_{p} / a=1.55$. The most striking feature of this figure is the large number of apparent resonances. Almost all of them are numerical artifacts, however, characterized by wild radial oscillations with wavelengths on the order of the grid size. The relatively broad peaks, however (labeled $a$ and $b$ on the figure) have smooth enough perturbed potentials to be candidates for real resonances. Peak $a$ has the character of a diocotron mode, while peak $b$ has more of the character of a plasma mode (the perturbed potential has two radial maxima). To try to make a more precise identification, we made a sequence of equilibria identical in every way except for their length. For each one we calculated the left- and right-hand sides of Eq. (37) and compared them to see if they tended to agree with each other as the plasma became long. The results of these calculations are displayed in Table I. Considering the crude way in which we made contact with Keinigs' calculation, the agreement is quite good (and perhaps even fortuitous). Nevertheless, based on the shape of the potential eigenfunction and on the approximate agreement with Keinigs' calculation, we feel 


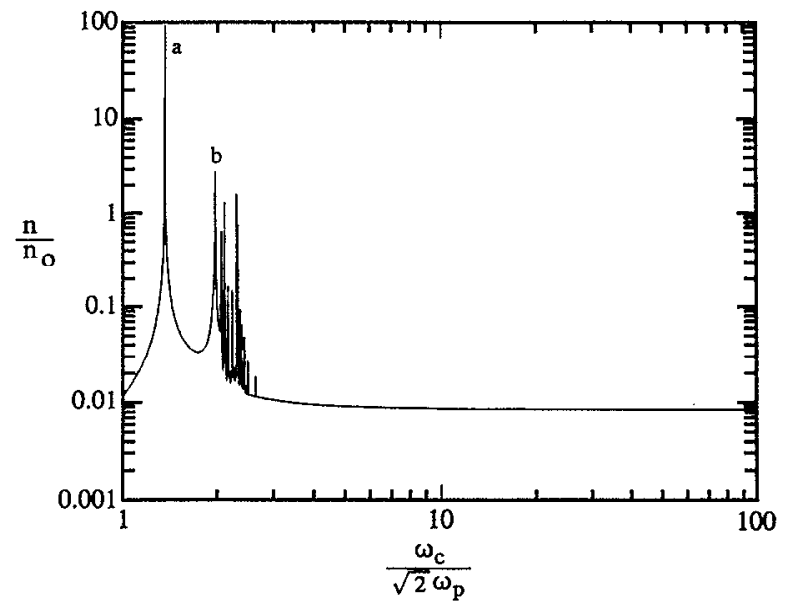

FIG. 6. Perturbed density for a thermal equilibrium with $r_{p} / a=0.5$ and $z_{p} / r_{p}=3$, where $z_{p}$ is the half-length of the plasma. The perturbed density is displayed versus magnetic field, expressed in terms of the Brillouin limit parameter $\omega_{c} \sqrt{2} \omega_{p}$. Most of the peaks are numerical artifacts, but peak $a$ is a diocotronlike resonance and peak $b$ is a plasmalike resonance.

confident that we have reproduced his calculation in full two-dimensional geometry. To make doubly sure, we tried varying the temperature of our equilibrium (Keinigs' calculation had $T=0$ ) to see if the resonance was sensitive to temperature. We dropped the temperature from $1 \mathrm{eV}$ down to $0.1 \mathrm{eV}$ and saw only a small $(3 \%)$ increase in the resonant value of the magnetic field. Since this resonance is perilously close to the Brillouin limit, we also tried to include some inertial effects perpendicular to the field by including, to first order in $\omega_{E} / \omega_{c}$, the inertial terms associated with the $\mathbf{E} \times \mathbf{B}$ drift in the perpendicular momentum equation. When the resonances were recalculated with these terms included we found that the resonant value of $\omega_{d} \sqrt{2} \omega_{p}$ for peak $a$ shifted upward by $20 \%$, while that for peak $b$ shifted up by $3 \%$. This means that inertial effects are important as the Brillouin limit is approached, but for longer plasmas where the resonances occur away from the Brillouin limit, they may be safely neglected. Hence we are fairly confident that we are able to calculate the diocotron and plasmalike resonances of two-dimensional plasmas in tilted magnetic fields with the numerical method described here.
TABLE I. Comparison between the left- and right-hand sides of Eq. (37) as the length of the plasma is changed. As the plasma gets long, the ratio of the two sides of the equation approaches one and the resonance moves farther from the Brillouin limit. In the longest plasma the two sides of the equation differ by less than $5 \%$. In all of these calculations, the plasma radius was one-half of the wall radius.

\begin{tabular}{|c|c|c|}
\hline$z_{p} / a$ & $\frac{\omega_{c}}{\sqrt{2} \omega_{p}}$ & $\frac{2 z_{p} / \pi a}{\omega_{d} \sqrt{2} \omega_{p}}$ \\
\hline 1.55 & 1.37 & 0.720 \\
\hline 2.29 & 1.79 & 0.816 \\
\hline 3.05 & 2.22 & 0.874 \\
\hline 3.80 & 2.65 & 0.913 \\
\hline 5.29 & 3.53 & 0.955 \\
\hline
\end{tabular}

\section{CONCLUSIONS}

We have found a simple perturbation expansion which allows us to calculate the linear response of a non-neutral plasma to a tilted magnetic field. The results of this calculation agree quite well throughout the bulk of the plasma with the previous three-dimensional equilibrium calculation. The largest disagreements come at the ends of the plasma where nonlinear contributions to the perturbed density are starting to become important. This expansion allows us to calculate the perturbed velocity in the plasma, but this is very small compared to the equilibrium flow and only produces deviations from the tilted field lines that are of the order of the $\alpha z$, concentrated at the ends of the plasma. We speculate that this flow might possibly cause mixing, and therefore increase the transport in such a plasma. We are also able to use the perturbation expansion to find the zero-frequency resonances discussed by Keinigs' and others in full two-dimensional geometry.

\section{ACKNOWLEDGMENTS}

The authors gratefully acknowledge helpful discussions with Lei Chen, Neil Rasband, and Kevin Fine, and especially thank one of the referees for making the suggestion which led to the calculations in Sec. IV.

'J. S. deGrassie and J. H. Malmberg, Phys. Rev. Lett. 39, 1077 (1972).

${ }^{2}$ C. F. Driscoll, Phys. Rev. Lett. 64, 645 (1990).

${ }^{3}$ J. H. Malmberg and C. F. Driscoll, Phys. Rev, Lett. 44, 654 (1980).

${ }^{4}$ D. J. Heinzen, J. J. Bollinger, F. L. Moore, W. M. Itano, and D. J. Wineland, Phys. Rev. Lett. 66, 2080 (1991).

5G. W. Hart, Phys. Fluids B 3, 2987 (1991).

${ }^{6}$ K. S. Fine, Ph.D. dissertation, University of California, San Diego, 1988.

${ }^{7}$ R. Keinigs, Phys. Fluids 24, 860 (1981). 